\title{
Genetic Algorithm for Optimization and Calibration in Process Simulation
}

\author{
T. Fühner, A. Erdmann, C.J. Ortiz, J. Lorenz \\ Fraunhofer Institute of Integrated Systems and Device Technology, \\ Schottkystrasse 10, 91058 Erlangen, Germany, fuehner@iisb.fraunhofer.de
}

\begin{abstract}
This paper proposes the use of genetic algorithms for process optimization and calibration of model parameters. The main principles of these evolution inspired optimizers are briefly explained. Afterwards, their application to two process simulation tasks is presented: (1) a layout problem in lithography processes and (2) the extraction of physical parameters of a diffusion model. The obtained results are shortly discussed.

\section{Introduction}

Optimization of processes or simulation parameters often requires the use of robust, global optimization methods. In many applications, very little a priori knowledge of the search space is available. Thus, constraining the search space is impossible or at least very time consuming. Furthermore, local search techniques are often not feasible as they strongly depend on an, often intricate, search for appropriate start values [1]. Genetic algorithms have been successfully applied to a wide range of different problems. A list of applications can be found elsewhere [2]. This paper proposes the use of genetic algorithms (GAs) as an alternative approach for optimization tasks in simulation. GAs are very robust and perform well even with noisy data, a problem often encountered in model calibration and process optimization applications. Moreover, with the proposed approach, optimization with virtually arbitrary numbers of variables and objectives can be performed fully automatically. We do not claim that the presented results cannot be obtained with other optimization techniques. The goal of this work is to demonstrated that GAs yield good, reproducable results for various process simulation problems.
\end{abstract}

\section{Principle of Genetic Algorithms}

GAs improve solutions by means of selection, recombination, and mutation [1]. GA operations are performed on bit-strings; in order to evaluate solutions, these bit-strings are decoded. Fig. 1(a) schematically demonstrates the flow of a genetic algorithm. In the first step initial solutions are randomly created. In a second step, solutions are evaluated according to a problem-specific objective function. The third step consists of a test for a pre-defined termination criterion (e.g., an acceptable fitness). In the fourth step pairs of individuals are selected according to their pay-off. The selected solutions are merged, yielding new solutions, in the fifth step (Fig. 1(b)). A random change on the individuals is optionally applied in the sixth step, in order to maintain a high level of diversity (Fig. 1(c)). The newly created solutions are evaluated thereafter, starting a new iteration step. 


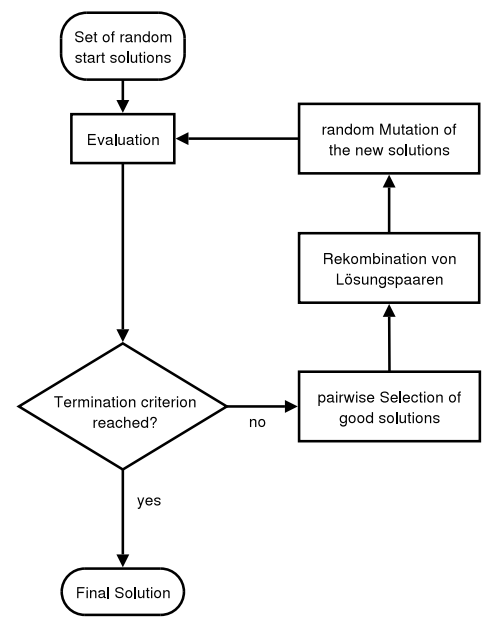

(a)

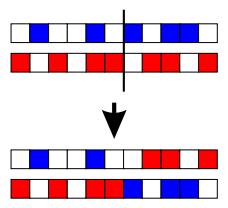

(b)

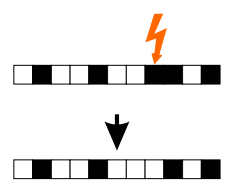

(c)

Figure 1: (a) Schematic flow of a genetic algorithm; (b) recombination: two parent solutions are merged yielding two offspring solutions; (c) mutation: random alteration of bits (genes).

\section{Application to Mask Optimization}

The performance of microelectronic integrated circuits strongly depends on the size of the smallest features on the chip. In the late nineties, the microelectronic industry started to employ various techniques that modified the geometry of the illumination and the mask in order to improve the resolution capability of processes. Imaging in this regime, however, is much more challenging, as the old WYSIWYG principle (What You See Is What You Get) is not valid anymore [3]. Thus, finding optimum illumination and mask geometries requires elaborate optimization techniques. This paper proposes the application of a genetic algorithm to improve the performance of lithographic processes by optimizing mask and source geometries. Merit or fitness functions are introduced that evaluate the imaging quality of arbitrary line patterns in a specified focus range. As a second criterion the manufacturability and inspectability of the mask are taken into account. All simulations have been conducted using the in-house lithography simulator of the Fraunhofer Institute IISB [4].

As an example, Fig. 2 illustrates the best geometry of a semidense lines/spaces mask after a specified number of optimization steps performed by the genetic algorithm. The lower row shows the corresponding process windows for the optimized mask (solid lines) in comparison to the process window of $110 \mathrm{~nm}$ dense lines/spaces (pitch $220 \mathrm{~nm}$, dashed lines). The goal of the optimization is to maximize the overlap between both process windows and to obtain a manufacturable design of the mask. After 10 iterations the mask consists of many small dark lines. The overlap between the process windows of dense and semidense features is rather small. After 50 generations a significant overlap of the process windows has been achieved. However, the high number of thin dark lines renders its production unfeasible. After about 800 iterations an appropriate mask layout has been obtained. The optimization took about 45 minutes using a 10 processor $(2.66 \mathrm{GHz}$ Pentium 4) computer cluster.

In this work the following optimization problems are investigated: the first was to find the 

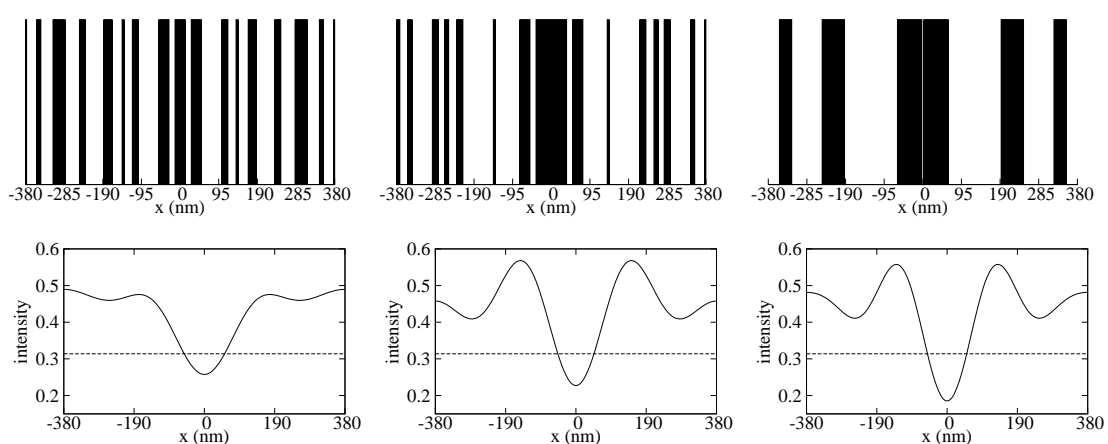

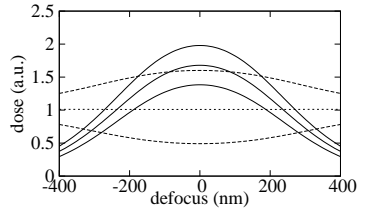

10th generation

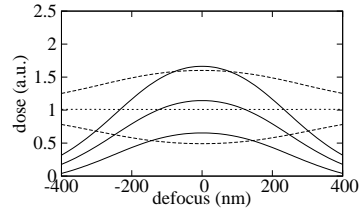

50th generation

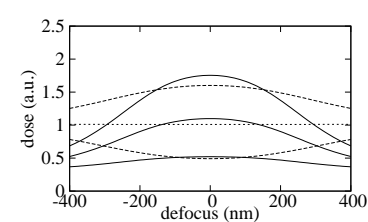

810th generation

Figure 2: Improvement of the image performance obtained with the GA for the discrete mask representation, upper row: mask geometry, center row: images at $250 \mathrm{~nm}$ defocus, lower row: process windows.

best lines/spaces proximity curves, that is, mask geometries and illumination parameters which guarantee optimum lithographic performance over a range of pitches from dense features ( pitch $=220 \mathrm{~nm}$ ) to almost isolated features (pitch $=1000 \mathrm{~nm}$ ). Afterwards, the optimization method is used to conduct an evaluation of different mask and source options. Finally, the optimization scheme is applied to 2D features (contact holes), yielding first promising results. A detailed discussions on the procedure and on results can be found elsewhere [5].

\section{Application to Calibration of Diffusion Model Parameters}

Self-interstitials generated by ion implantation aggregate during subsequent thermal anneals into extended defects [6]. These maintain a non-equilibrium self-interstitial supersaturation in their vicinity which, in turn, enhances the diffusivity of dopants such as boron. For the simulation and optimization of annealing steps physical and well calibrated models for these reactions are essential. In the current work we implemented a one-dimensional model accounting for the capture and emission of interstitials into the PDE solver PROMIS 1.5. The simulator was then linked to the GA to optimize values for the formation energies of small interstitial clusters (with size $<10$ ), the stackingfault energy of $\{113\}$ defects, and the self- diffusion coefficient $D_{I} C_{I}^{*} / C_{\mathrm{Si}}$ of silicon via self-interstitials at different temperatures. Different kinds of experimental data were combined for the optimization with the GA. Fig. 3 shows experimental data $[7,8]$ for the supersaturation of interstitials which is the key parameter for transient enhanced diffusion. The dashed lines show the excellent agreement for temperatures of 600 and $800^{\circ} \mathrm{C}$ between simulations and those experiments which were used for the optimization with the GA. The optimization time was about three days on a single processor (DEC Alpha $666 \mathrm{MHz}$ ) machine.

Furthermore, Fig. 3 clearly evidences that our model is able to predict independent experimental data obtained at 700 and $740{ }^{\circ} \mathrm{C}$, demonstrating the predictivity of the model as well as the consistency of the optimized parameter set. Whereas this paper discusses 


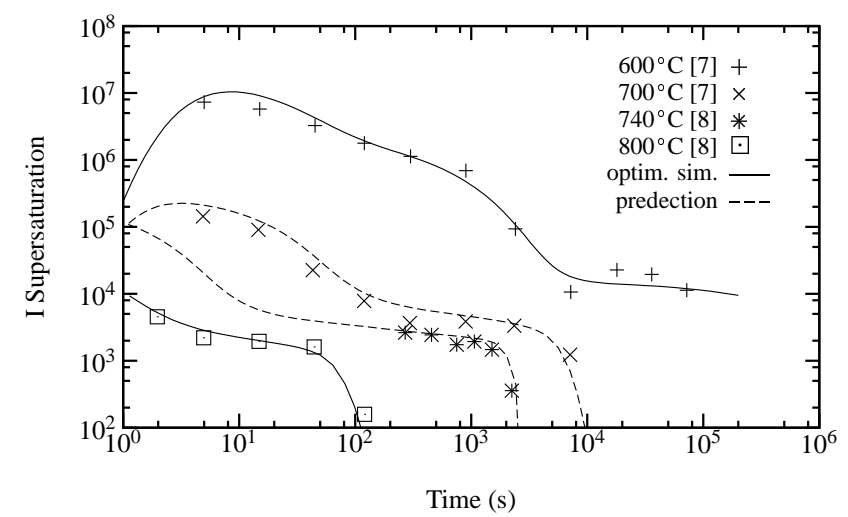

Figure 3: Comparison between experimental supersaturation and simulations with the optimized parameter set: Simulation of data used during optimization (dashed lines) and prediction of complementary data (solid lines).

the benefits of the GA, a detailed discussion of the experiments and the physical model is being given elsewhere [9].

\section{Conclusions}

A genetic algorithm has been presented which is widely applicable to optimize parameters of physical models, process parameters, and mask design.

\section{Acknowledgements}

Part of this work has been carried out within the project FRENDTECH (IST project 2001-30129), funded by the European Commission.

\section{References}

[1] D. E. Goldberg. Genetic Algorithms in Search, Optimization, and Machine Learning. AddisonWesley, Reading, MA, 1989.

[2] T. Bäck, F. Hoffmeister, and H.P. Schwefel. Applications of evolutionary algorithms - 4th extended edition. Technical Report SYS-2/92, University Dortmund, 1993.

[3] A. K. Wong. Resolution Enhancement Techineques in Optical Lithography. In Tutorial Texts in Optical Engineering, TT47. SPIE Press, 2001.

[4] A. Erdmann and W. Henke. Simulation of optical lithography. In Optics and Optoelectronics - Theory, Devices and Applications, Proc. SPIE 3729, page 480, 1999.

[5] T. Fühner, A. Erdmann, R. Farkas, B. Tollkühn, and G. Kókai. Genetic algorithms to improve mask and illumination geometries in lithographic imaging systems. In Raidl et al., editor, EvoWorkshops 2004, pages 208-217, 2004.

[6] A. Claverie, B. Colombeau, B. de Mauduit, C. Bonafos, X. Hebras, G. Ben Assayag, and F. Cristiano. Extended defects in shallow implants. Appl. Phys., A 76:1025, 2003.

[7] N.E.B. Cowern, G. Mannino, P. A. Stolk, F. Roozeboom, H. G. A. Huizing, J. G. M van Berkum, F. Cristiano, A. Claverie, and M. Jaraiz. Energetics of Self-Interstitial Clusters in Si. Phys. Rev. Lett., 82(22):4460, 1999.

[8] F. Cristiano, P. Calvo, and N. Cherkashin. private communication.

[9] C.J. Ortiz P. Pichler, T. Fühner, F. Cristiano, A. Claverie, B. Colombeau, and N. E. B. Cowern. A physically-based model for the spatial and temporal evolution of self-interstitial agglomerates in ion-implanted silicon. J. Appl. Phys, 2004. submitted. 